\title{
Herd prevalence of bovine brucellosis and analysis of risk factors in cattle in urban and peri-urban areas of the Kampala economic zone, Uganda
}

\author{
Kohei Makita ${ }^{1,5^{*}}$, Eric M Fèvre ${ }^{2}$, Charles Waiswa ${ }^{3}$, Mark C Eisler ${ }^{1}$, Michael Thrusfield ${ }^{4}$ and Susan C Welburn ${ }^{1}$
}

\begin{abstract}
Background: Human brucellosis has been found to be prevalent in the urban areas of Kampala, the capital city of Uganda. A cross-sectional study was designed to generate precise information on the prevalence of brucellosis in cattle and risk factors for the disease in its urban and peri-urban dairy farming systems.

Results: The adjusted herd prevalence of brucellosis was 6.5\% (11/177, 95\% Cl: 3.6\%-10.0\%) and the adjusted individual animal prevalence was 5.0\% (21/423, 95\% Cl: 2.7\% - 9.3\%) based on diagnosis using commercial kits of the competitive enzyme-linked immunosorbent assay (CELISA) for Brucella abortus antibodies. Mean within-herd prevalence was found to be $25.9 \%$ (95\% Cl: $9.7 \%$ - 53.1\%) and brucellosis prevalence in an infected herd ranged from $9.1 \%$ to $50 \%$. A risk factor could not be identified at the animal level but two risk factors were identified at the herd level: large herd size and history of abortion. The mean number of milking cows in a free-grazing herd (5.0) was significantly larger than a herd with a movement restricted (1.7, $p<0.001)$.
\end{abstract}

Conclusions: Vaccination should be targeted at commercial large-scale farms with free-grazing farming to control brucellosis in cattle in and around Kampala city.

\section{Background}

Brucellosis remains one of the world's most widespread zoonoses [1]. The disease in humans, known as 'undulant fever', 'Mediterranean fever' or 'Malta fever' [2], remains an important public health problem. On genetic grounds the Brucella grounp can be regarded as variants of a single species, Brucella melitensis, however for practical reasons, six main species are distinguished: $B$. abortus, $B$. suis, B. melitensis, B. neotomae, B. ovis and B. canis [2]. Historically, only B. abortus, B. suis and B. melitensis have been considered as zoonotic pathogens but recent reports have shown that the newly recognised marine mammal species also have zoonotic potential $[3,4]$. Out of these zoonotic Brucella species, bulk of human diseases is caused by B. abortus and B. melitensis [4]. Brucellosis in cattle (mainly caused by Brucella abortus) poses not only a significant threat as a source of infection to humans but also

\footnotetext{
* Correspondence: kmakita@rakuno.ac.jp

${ }^{1}$ Centre for Infectious Diseases, Division of Pathway Medicine, School of Biomedical Science, College of Medicine and Veterinary Medicine, The University of Edinburgh, 1 Summerhall Square, Edinburgh, EH9 1QH, UK Full list of author information is available at the end of the article
}

the risk of economic losses. Losses through abortion or calf death is a huge economic constraint for farmers [5] and establishment of the carrier state in a large proportion of animals may lead to a $20 \%$ reduction in the milk yield [6]. In areas where culling or other means of brucellosis control are not practised, long-term chronic infections are often associated with carpal hygromas and infertility [7]. Aborted foetuses and discharges contain large numbers of infectious organisms, and chronically infected cattle can shed lower numbers of organisms via milk and reproductive tract discharges, and can also vertically transmit infection to subsequently born calves, thereby maintaining disease transmission [7].

Human brucellosis has been found to be prevalent in urban areas of Kampala, Uganda [8]. The sources of the risks from informally-marketed milk and the effective control measures for human brucellosis have been described. Constructing boiling centres either in dairy production areas or peri-urban Kampala and enforcing traders to sell to these centres would reduce the risk the most [9]; however control of brucellosis in the source cattle would reduce the risks to humans most effectively. It is therefore 
important to know the prevalence and risk factors in cattle. A number of studies of brucellosis prevalence in cattle have been conducted in Uganda [10-15]. It has been suggested that the high plateau lands of western and eastern Uganda were zones of hyper-endemicity, for both human and bovine brucellosis, while the Central and Southern part of the Uganda along the shores of Lake Victoria were zones of moderate endemicity [16]. Brucellosis is also prevalent among the Ugandan wildlife population [17].

B. melitensis is normally associated with goats and sheep but can cause cross-species infection with dairy herds [2]. In eastern and western Uganda, 13\% (12/93) of goat herds had goats with positive reaction in both the brucellosis card test and the $B$. melitensis tube agglutination test [18]; the risk of human and bovine brucellosis due to B. melitensis is not negligible in Kampala.

Recent studies in Uganda have shown that differences in disease prevalence in cattle are associated with different production systems $[13,19]$. In zero-grazing systems (for example, in Eastern Uganda) where there is a low level of herd-to-herd contact, the herd-level prevalence was low (5.5\%) while in pastoral systems (for example, in Central Uganda) where there are high level of herd-toherd contact, the prevalence was 100\% [19]; this finding is supported across studies of brucellosis in sub-Saharan Africa [7].

The present study attempts to elucidate the prevalence of brucellosis, as well as risk factors for brucellosis in cattle in urban and peri-urban areas of Kampala, providing inputs for evidence-based disease control in Uganda.

\section{Results}

\section{Prevalence of brucellosis}

In total, 423 secondary sampling units (cows) in 177 (out of 625) primary sampling units (cattle herds) were sampled; the actual sample fraction at herd level was $28.3 \%$ (177/625). Out of 177 sampled herds, 11 herds were found to be positive showing an adjusted herd-level prevalence of brucellosis of $6.5 \%$ (95\% CI: 3.6-10.0). At the individual animal level, 21 of 423 cows were positive with the competitive enzyme-linked immunosorbent assay (CELISA) and the adjusted prevalence was 5.0\% (95\% CI: $2.7-9.3)$.

\section{Herd size}

Figure 1 shows the frequency of herds according to the number of milking cows in a herd. The geometric mean [20] of number of cows per farm was 1.7. Six large herds with more than or equal to 10 milking cows were located in peri-urban and rural areas; however even in these areas, the majority of herds were small-scale, comprising less than 10 cows. The maximum herd size was 24 cows among all the 177 farms (a farm is equivalent to a herd in the present paper) and for the herds with less than or

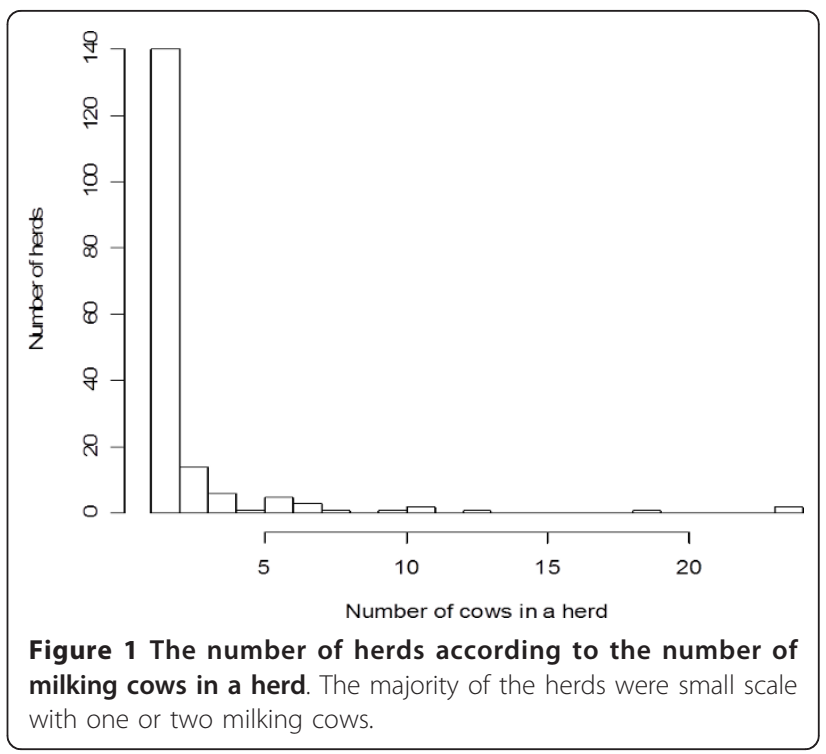

equal to 24 animals, the sample size in a herd was always calculated as all the cows belonged to; sera were collected from all the milking cows in the selected farms.

\section{Within herd prevalence}

Table 1 shows the proportions of brucellosis positive cows according to the number of cows in a herd. Within herd prevalence of brucellosis among infected herds varied between 9.1 to $50.0 \%$, and the herd level information-weighed overall mean within-herd prevalence was $25.9 \%$ (95\% CI: 9.7\% - 53.1\%). There was no relationship between within-herd prevalence and herd size; the logit of within herd prevalence of brucellosis did not change with the number of milking cows in a herd (slope $=-0.019$, se $=0.03, p=0.652$ ).

\section{Risk factors for brucellosis at the animal level}

No significant risk factor for brucellosis was detected, by univariate analysis, at the animal level. Neither the use of a bull for insemination, nor a history of vaccination against brucellosis nor any history of abortion were found to be either significant risk or preventive factors for brucellosis infections at the animal level (all $p$-values of prevalence ratios $[21,22]$ were more than 0.05 , see Table 2). Furthermore, at the animal level, the mean rank of body condition score was also not found to be significantly different between CELISA positive (median $=3.0$, average rank $=218.2)$ and negative cows (median $=3.0$, average rank $=211.7, \mathrm{df}=1, p=0.799$ ). The mean parity was not significantly different between CELISA positive (2.76, 95\%CI: 2.06 - 3.69) and negative cows (2.39, 95\% CI: $2.24-2.56, p=0.341$ ) and the mean age was also not found to be significantly different between CELISA positive (5.53) and negative cows (5.20, $p=0.420)$. 
Table 1 The number of herds with infected cows and within-herd prevalence according to the number of cows in a herd

\begin{tabular}{|c|c|c|}
\hline Number of cows in a herd & Number of herds with infected cows & Within-herd prevalence (\%) \\
\hline 2 & 3 & $50(50)^{*}$ \\
\hline 4 & 1 & 25 \\
\hline 5 & 1 & 20 \\
\hline 6 & 1 & 16.7 \\
\hline 7 & 1 & 14.3 \\
\hline 11 & 1 & 9.1 \\
\hline 13 & 1 & 15.4 \\
\hline 19 & 1 & 15.8 \\
\hline 24 & 1 & 25 \\
\hline
\end{tabular}

*Mean and range ().

\section{Risk factor of brucellosis at the herd level}

In the univariate analysis, large herd size was associated with sero-positive status of herds; the number of milking cows in a herd was significant larger in sero-positive herds (8.6) than sero-negative herds $(2.0, p<0.001)$. Three factors-free-grazing farming, a history of vaccination and a history of abortion - had $p$-values less than 0.2 [23] in prevalence ratios (see Table 3). There was no collinearity between each two of these four factors.

Two factors: large herd size (OR: 1.3 (95\% CI: 1.1-1.5), $p<0.001$ ) and history of abortion (OR: 4.1 (1.0-17.6), $p=$ 0.059 , it remained because of the biological plausibility) remained in the final model and the model passed the goodness-of-fit test, Hosmer-Lemeshow test [24] (sum of square $=53.1, \mathrm{df}=167, p=0.60)$. Although the factor, free-grazing farming (OR: $2.7, p=0.2$ ) did not remain, its removal changed the logit of herd size by $19.8 \%((0.26-$ $0.217) / 0.217$, data not shown in a table), and by a GLM with quasipoisson errors, the mean number of milking cows in a free-grazing herd (5.0) was significantly larger than a herd with the movement of cows restricted (1.7, p $<0.001)$. This suggested that a risk factor, being large herd, was associated with free-grazing of cattle herds.

\section{Discussion}

In the present study, a brucellosis herd prevalence and individual animal prevalence were observed in urban and peri-urban areas of Kampala using a probability sampling framework and a highly sensitive and specific diagnostic test: the CELISA. A previous study of only 16 farms (one in Kampala, five in Mukono and 10 in Wakiso) in central and southern parts of Uganda reported a high prevalence of brucellosis at the herd level $(56.3 \%, 9 / 16)$ and at animal level, 5.0\% (19/383) using both the Rose Bengal Test (RBT) and serum agglutination test (SAT) [15]. In a study of 245 cattle serum samples from urban and peri-urban areas of Kampala, 42\% were positive for antibodies against Brucella spp. using the slow serum tube agglutination test [14]. However, both the SAT and RBT are less specific than the CELISA [25] and the reported high prevalence (s) at herd level might be due to false-positive serum reactions (FPSR) and or due to bias in farm selection. False-positive serum reactions in Brucella spp. screening tests are known to be caused by unrelated Enterobacteriaceae [26-35] and CELISA can eliminate such reactions [33]. B. abortus vaccination strain 19 also gives rise to an antibody response similar to that resulting from natural infection [33] but CELISA can eliminate this falsepositive reaction only by approximately $50 \%$ [36]. In our study, 9/177 (5.1\%) of farms held vaccinated cattle and the CELISA positive reactions of vaccinated cows in two farms might be due to the false-positive reactions with B. abortus vaccination strain S19.

Table 2 Univariate analysis for brucellosis at the animal level

\begin{tabular}{|c|c|c|c|c|c|}
\hline Factors & $\begin{array}{c}\text { Diseased } \\
\text { animals }\end{array}$ & $\begin{array}{l}\text { Healthy } \\
\text { animals }\end{array}$ & $\begin{array}{c}\text { Prevalence } \\
(\%)\end{array}$ & $\begin{array}{l}\text { Prevalence } \\
\text { ratio }\end{array}$ & $p$-value \\
\hline \multicolumn{6}{|l|}{ Insemination } \\
\hline Bull & 18 & 310 & 5.5 & 1.74 & 0.434 \\
\hline $\mathrm{Al}$ & 3 & 92 & 3.2 & & \\
\hline \multicolumn{6}{|l|}{ Vaccination } \\
\hline Vaccinated & 5 & 60 & 7.7 & 1.72 & 0.345 \\
\hline Not vaccinated & 16 & 342 & 4.5 & & \\
\hline \multicolumn{6}{|l|}{ Abortion } \\
\hline Aborted & 3 & 42 & 6.7 & 1.40 & 0.479 \\
\hline Not aborted & 18 & 360 & 4.8 & & \\
\hline
\end{tabular}


Table 3 Univariate analysis for brucellosis at the herd level

\begin{tabular}{|c|c|c|c|c|c|}
\hline Factors & Infected herds & Healthy herds & Prevalence (\%) & Prevalence ratio & $p$-value \\
\hline \multicolumn{6}{|l|}{ Urbanicity } \\
\hline Urban & 4 & 50 & 7.4 & $x^{2}=0.59^{*}$ & 0.743 \\
\hline Peri-urban & 2 & 47 & 4.1 & $d f=2$ & \\
\hline Rural & 5 & 69 & 6.8 & & \\
\hline \multicolumn{6}{|l|}{ Free-grazing } \\
\hline Free-grazing & 7 & 26 & 21.1 & 6.15 & $<0.001$ \\
\hline Restricted & 4 & 140 & 2.8 & & \\
\hline \multicolumn{6}{|l|}{ Breed } \\
\hline Improved & 4 & 57 & 6.6 & $x^{2}=0.47^{*}$ & 0.790 \\
\hline Cross & 3 & 61 & 4.7 & $d f=2$ & \\
\hline Indigenous & 4 & 48 & 7.7 & & \\
\hline \multicolumn{6}{|l|}{ Insemination } \\
\hline Bull & 8 & 121 & 6.2 & 0.90 & 1 \\
\hline $\mathrm{Al}$ & 3 & 45 & 6.3 & & \\
\hline \multicolumn{6}{|l|}{ Vaccination } \\
\hline Vaccinated & 2 & 7 & 22.2 & 3.76 & 0.10 \\
\hline Not vaccinated & 9 & 159 & 5.4 & & \\
\hline \multicolumn{6}{|l|}{ Abortion } \\
\hline Aborted & 4 & 21 & 16.0 & 3.06 & 0.052 \\
\hline Not aborted & 7 & 145 & 4.6 & & \\
\hline \multicolumn{6}{|l|}{ Bought-in cattle } \\
\hline Yes & 7 & 119 & 5.6 & 0.61 & 0.716 \\
\hline No & 3 & 40 & 7.0 & & \\
\hline \multicolumn{6}{|l|}{ Persistent fever } \\
\hline Exist & 1 & 16 & 5.9 & 0.86 & 1 \\
\hline Not exist & 10 & 150 & 6.3 & & \\
\hline
\end{tabular}

* Likelihood ratio test result

The present study identified two risk factors for brucellosis at the herd level: large herd size and history of abortion. For the correct causality, the result should be interpreted that Brucella sero-positive status of herds was a risk factor of abortion. Free-range farming did not remain in the final multivariable model; however being a large herd was associated with a free-range farming. Moreover, the moderate change of logit of the factor - large herd, by a removal of a factor - free-grazing from a model suggested a confounding to large herd by free-grazing which can maintain or enhance infection with brucellosis in a herd. Free-grazing farming and abortion have been previously identified as risk factors relating to pastoral systems $[7,19,37]$. The unique characteristic observed in periurban areas of Kampala was the presence of commercial large-scale free-grazing farming within a fenced zone, although most farms are small-scale. This commercialized management system, including milk sales and animal health inputs e.g. periodical insecticide spraying, is also observed in the Mbarara dairy production area and could be distinguished from zero-grazing systems (improved breed) and communal-grazing systems (local and cross breeds) which are dominant in peri-urban Kampala. These commercial herds rarely come into contact with any other herds because they are separated by a physical barrier (a fence) but the transmission of brucellosis can be maintained within each large herd while grazing.

In the present study, a history of bought-in cattle was not a risk factor for brucellosis, suggesting the endemic status of brucellosis in and around Kampala may be maintained indefinitely by low-level within herd transmission. Use of bulls was shown not to be a risk factor. It might be also because of the endemic status; however two zero-grazing herds using artificial insemination were found to be infected with brucellosis in this study. Purchase of infected animals or contamination of frozen semen with Brucella could not be ruled out [6].

No risk factors for brucellosis at the level of an individual animal were identified in the present study. In Mbarara, previous studies have shown that keeping exotic breeds [13] was a risk factor but this was not found to be a risk factor in the present study. Age of animal has also been shown to be a risk factor in other studies in Mbarara, Uganda [13] and also in Northern Ireland [38]. 
While parity and age were not identified as risk factors in the present study, this may be simply because all samples were taken only from milking cows which are usually more than two years old.

The level of urbanisation in this study was not a risk factor for brucellosis in Kampala but large herds with free-grazing farming (which are risk factors) are located in peri-urban areas, and the Ugandan Veterinary Authorities should consider these areas for practical control programmes in and around Kampala, with a special focus on large farms with free-grazing farming.

\section{Conclusions}

The present study found three risk factors for brucellosis in cattle in the urban and peri-urban areas of Kampala: being large herd, free-grazing farming and history of abortion. Vaccination remains the most appropriate control measure in Uganda because brucellosis is endemic and stamping out may be economically too burdensome. Vaccination campaigns, especially focusing on large free-grazing herds in the peri-urban areas, could significantly reduce the prevalence of brucellosis in cattle in the areas and reduce the risk of transmission to humans.

\section{Methods}

\section{Study sites}

The urban and peri-urban areas of Kampala are semihumid areas with mean annual rainfall of $1261 \mathrm{~mm}$ between 1999 and 2005 (standard deviation: 197) [39]. Common production systems are zero-grazing in urban and mixed crop-livestock systems in peri-urban and rural areas. Zero-grazing is also practised in peri-urban areas. Communal grazing, tended by herdsmen, is common in peri-urban areas and rural areas close to the peri-urban areas. A small number of large-scale dairy farms with fenced large grazing yards are also seen in these areas. All these systems were represented in the herds sampled in this study.

Uganda has an administrative system comprising five levels: District (called as Local Council 5), County (LC4), Sub-County (LC3), Parish (LC2) and zone/village (LC1) [40].

This study was conducted in 56 cattle-keeping LC1 units in urban (29 LC1s), peri-urban (11 LC1s), and rural areas (16 LC1s) of the Kampala economic zone in Uganda (see Figure 2). Stratified random sampling was used; strata were LC3s (Sub-counties) and sampling units were LC1s. LC3s where more than half of the area is located between five and $20 \mathrm{~km}$ from Nakasero, the political and economic centre of Kampala city, were selected for the study. The numbers of LC1s in each stratum were assigned using proportional allocation [41]. Eighty seven LC1s were selected from 790 LC1s in the 10 LC3s. Three LC3s in the central part of Kampala District were excluded from the selection because more than half of the areas were located within $5 \mathrm{~km}$ from Nakasero. LC1 leaders and residents were interviewed and the $87 \mathrm{LC} 1 \mathrm{~s}$ were classified into urban, peri-urban and rural, based on a decision-tree model as previously described [42]. Of these LC1s, non-residential LC1s (e.g. universities and institutions) and LC1s without cattle were excluded. In the final analysis, $56 \mathrm{LC} 1 \mathrm{~s}$ with cattle herds/individuals were selected.

\section{Ethical statement}

This study involves an investigation using interviews with farmers as well as blood sampling from cattle. The study protocol was assessed and approved by the Uganda National Council for Science and Technology (UNCST) on 14th September 2005, with its reference number A 432.

\section{Multi-stage Sampling framework \\ (1) Sample size of primary sampling units}

Primary sampling units (cattle herds) were calculated based on a census of the total number of cattle herds in the selected $56 \mathrm{LC} 1 \mathrm{~s}$, obtained from interviews with LC1 Leaders [42]. WinEpiscope 2.0 [43] was used to calculate sample size for prevalence estimates. The expected herd prevalence was set to $55.6 \%$ based on the brucellosis herd prevalence in Mbarara [13], with an accepted error and level of confidence selected as $\pm 5 \%$ and $95 \%$ respectively. The sampling fraction (24\%) that is, the percentage of herds to sample among total herds in the $56 \mathrm{LC} 1 \mathrm{~s}$ was calculated by dividing the calculated sample size by total number of herds estimated from above interviews. This sample fraction (24\%) was used to determine the sample size of cattle herds in each LC1 at the sampling based on the complete list of cow-keeping farmers prepared by the LC1 Leader in advance.

\section{(2) Sample size of secondary sampling units}

Milking cows, including cows in the dry period, were selected as secondary sampling units. Bulls, calves and heifers were excluded from the present study because the focus was on cows as potential sources of infected milk. Since milk from different cows is usually mixed at the farm before selling, each farm should be judged as either infected with Brucella or not, by determining whether a farm is free from the disease, or has at least one serologically positive cow. To determine the sample size in the field, a card with the sample size of cows for disease detection with the probability of causing error less than $5 \%$ in different farm sizes was prepared using FreeCalc version 2 (Australian Veterinary Animal Health Services). Sensitivity and specificity were entered as $95.4 \%$ and $99.9 \%$ respectively as an imperfect test, buffered antigen plate agglutination test (BPAT) (sensitivity 0.954 , specificity 0.977 ) 


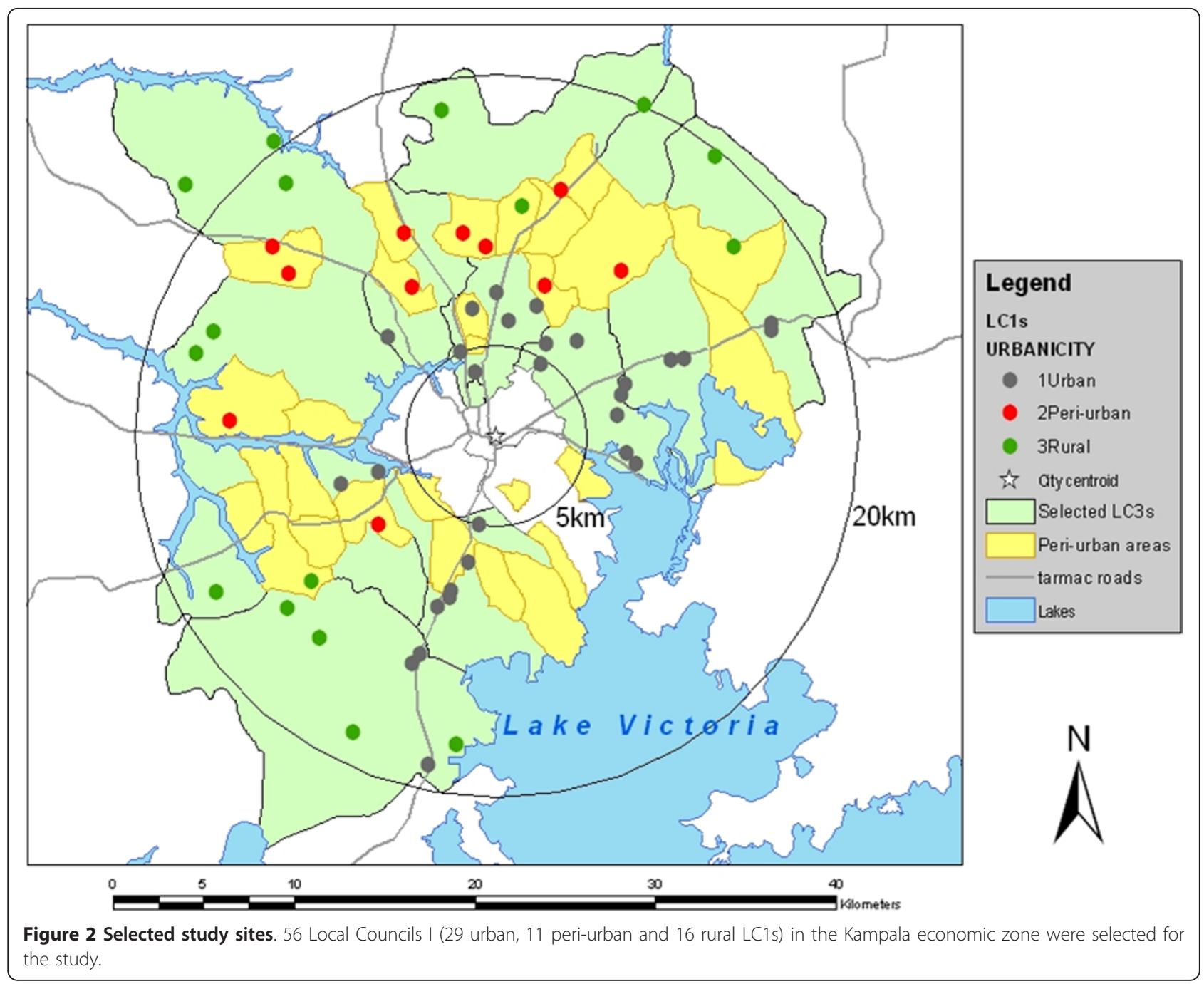

[44], was initially to be used for the present study complemented by competitive enzyme-linked immunosorbent assay (CELISA, sensitivity 1.000, specificity 0.999) [33]; however CELISA was used for all the samples to increase the accuracy of the estimation. Estimated cattle prevalence at individual animal level for the calculation was selected as $5 \%$, in order to test conservatively that the farm is free from brucellosis - a lower value than that used in other similar studies (central and southern parts of Uganda were found to have $8-16 \%$ of herds positive [15], and in Mbarara, herd prevalence was 55.6\% [13].

\section{Herd selection}

Sample herds were selected in each LC1 on the basis of random sampling from the list of all cow-keeping farms. Any listed cattle farms that did not have a cow were excluded from the list of cattle herds before herd selection. Prior to sampling, verbal consent was given by each farmer. When permission for sampling or interview was declined, another herd was selected from the listed herds on the basis of random sampling.

\section{Cattle sampling - Interviews with farmers}

Cattle sampling was conducted during October and November 2007. Cattle owners were interviewed for information about their farms and milking cows using a questionnaire. Data from interviews and diagnostic tests were digitized using Microsoft Access (Microsoft Office $\mathrm{XP}$, Redmond, USA). A nine-point body condition score, using half-point increments from 1 to 5 [45], was recorded for all sampled cows.

\section{Collection of blood samples}

Blood was taken from either the jugular or sacral medial vein of cows using 21 gauge needles and disposable $5 \mathrm{ml}$ plastic syringes. Blood was kept in plain vacuum plastic tubes (Vacutainer ${ }^{\circledR}$ ) and left for 30 minutes to 1 hour at ambient temperature to separate serum from the blood 
clot. Serum was collected from the Vacutainer using a disposable plastic Pasteur pipette, dispensed to an Eppendorf tube and stored in a cool-box in the field. Eppendorf tubes were then stored in the freezer at $-20^{\circ} \mathrm{C}$.

\section{Serological tests}

CELISA kits were purchased from the Veterinary Laboratories Agency (Surrey, UK). Kits were sent directly to Uganda, maintaining the cold chain and, immediately after receipt, were kept refrigerated at the Central Laboratory of the Department of Veterinary Medicine, Faculty of Veterinary Medicine, Makerere University, as instructed by the suppliers. CELISA was performed following the manufacturer's protocol at the Molecular Laboratory, Department of Molecular Biology, Faculty of Veterinary Medicine, Makerere University.

\section{Statistical analysis}

Test prevalence was regarded as the true prevalence because sensitivity and specificity of CELISA were 1.000 and 0.999 respectively [33]. As the present study used multi-stage sampling and intra-class correlations at the levels of LC3, LC1 and farm can affect the variance of the estimated prevalence, robust variance estimation [23] was performed using Generalized Estimating Equation (GEE) [46] in statistical software R version 2.13.1. The levels which correlations were controlled were LC3 and LC1 for the herd prevalence, and LC3, LC1 and farm for the animal prevalence. For the animal prevalence estimation, the numbers of cows sampled in the farms were served as offsets to weigh the inter-farm variance and the GEE was performed with Poisson errors.

The geometric mean was used to calculate the mean herd (farm) size because there were some outliers and the data error structures were not Normally distributed.

Within-herd prevalence and the $95 \%$ confidence inter$\mathrm{val}$, and the relationship between within herd prevalence and herd size (number of cows per farm) were analyzed using a GLM with binomial errors using R.

For risk factors for brucellosis at the animal level, univariate analysis was used. Prevalence ratios were calculated for use of bull for insemination, history of vaccination against brucellosis, and history of abortion using unconditional maximum likelihood estimation for the risk ratio and Fisher exact p-values in EpiTool version 0.5-6 [47] in R. Body condition score was compared between CELISA positive and negative cows using Kruskal-Wallis Rank Sum Test in R. Mean numbers of births and mean age was compared between CELISA positive and negative cows using a One-Way ANOVA after log-transformation with $\mathrm{R}$ because the transformation parameter $\lambda$ (lambda) in Box-Cox transformation [48] statistic was close to zero.

For risk factors for brucellosis at the herd level, univariate analysis was performed. The number of milking cows in a herd- herd size- was compared between seropositive herds and sero-negative herds using GLM with quasipoisson errors as data were overdispersed. Prevalence ratios were calculated for farming style (free-grazing and movement-restricted farming: tethered and zero-grazing), insemination (use of artificial insemination (AI) or bull), history of vaccination against brucellosis, history of abortion, bought-in sampled cows, and existence of family member or cattle keeper with persistent fever using EpiTools in R. For categorical data: level of urbanisation (urban, peri-urban and rural) and cattle breed, GLMs with binomial errors with the predictors were compared with the GLMs without the predictors by likelihood ratio tests in $\mathrm{R}$.

Factors at the herd level with the p-value less than 0.2 [23] in univariate analysis were investigated further. The factors which do not have collinearity with other factors (correlation coefficient less than 0.9) were fed into a multivariable logistic regression model. Step-wise model simplification was performed checking with a likelihood ratio test. The final model was diagnosed for goodness-of-fit using Hosmer-Lemeshow test [24] in LDdiag [49] in R. Confounding was tested monitoring the change of logit of factors by removing a suspected factor from the model [20], and association between the factors suspected for a confounding was tested where necessary.

\section{Acknowledgements and Funding}

We are grateful to the UK Department for International Development (DFID) Animal Health Programme (KM, EMF, MCE, CW, SCW) for support for the research undertaken in this study (although the views expressed are not necessarily those of DFID). KM thanks the Japan International Cooperation Agency (JICA) for research grant support for this study. Diagnostic tests were performed by Monica Namayanja and Steven Odongo at Makerere University. CW and SCW are supported by ICONZ, EU FP7 and DFID Research Into Use Programme. EMF is supported by the Wellcome Trust (085308). The cattle farm survey was greatly facilitated by Emilian Ahimbisibwe, Moses Mwanja and Herbert Mutumba, the Kampala, Wakiso and Mukono District Veterinary Officers and their very helpful and hard working colleagues. We thank lan Handel and Mark Bronsvoort at the University of Edinburgh for useful statistical discussions. We would also like to thank the Sub-County Chairmen, LC1 leaders and residents and dairy farmers who participated in this study.

\section{Author details}

${ }^{1}$ Centre for Infectious Diseases, Division of Pathway Medicine, School of Biomedical Science, College of Medicine and Veterinary Medicine, The University of Edinburgh, 1 Summerhall Square, Edinburgh, EH9 1QH, UK. ${ }^{2}$ Centre for Infectious Diseases, Ashworth Laboratories, The University of Edinburgh, Kings Buildings, Edinburgh, EH9 3JT, UK. ${ }^{3}$ Faculty of Veterinary Medicine, Makerere University, P.O. Box 7062, Kampala, Uganda. ${ }^{4}$ Veterinary Clinical Sciences, Royal Dick School of Veterinary Studies, College of Medicine and Veterinary Medicine, The University of Edinburgh. EH25 9RG, UK. ${ }^{5}$ Current Address: School of Veterinary Medicine, Rakuno Gakuen University, Ebetsu, 069-8501, Japan, and Improving Marketing Opportunities Theme, International Livestock Research Institute, PO Box 30709, Nairobi, Kenya.

\section{Authors' contributions}

Conceived and designed the study: KM EMF CW MCE MT \& SCW. Conducted field surveys: KM CW. Analyzed the data: KM. Wrote the paper: KM EMF SCW. All authors read and approved the final manuscript. 
Received: 4 March 2011 Accepted: 18 October 2011

Published: 18 October 2011

\section{References}

1. WHO: Seven neglected endemic zoonoses - some basic facts.[http:// www.who.int/zoonoses/neglected_zoonotic_diseases/en/index.html].

2. WHO: Brucellosis in humans and animals World Health Organization; 2006.

3. Sohn AH, Probert WS, Glaser CA, Gupta N, Bollen AW, Wong JD, Grace EM, McDonald WC: Human neurobrucellosis with intracerebral granuloma caused by a marine mammal Brucella spp. Emerg Infect Dis 2003, 9:485-88.

4. Franco MP, Mulder M, Gilman RH, Smits HL: Human Brucellosis. Lancet Infect Dis 2007, 7:775-86.

5. Birley MH, Lock K: The Health Impacts of Peri-urban Natural Resource Development Liverpool School of Tropical Medicine; 1999

6. Aiello SE, Mays A, (Eds): The Merck Veterinary Manual, Eighth Edition Whitehouse station, NJ, USA: Merck \& CO., Inc; 1998.

7. McDermott JJ, Arimi SM: Brucellosis in sub-Saharan Africa: epidemiology, control and impact. Vet Microbiol 2002, 90:111-134.

8. Makita K, Fèvre EM, Waiswa C, Kaboyo W, Bronsvoort BMDC, Eisler MC, Welburn SC: Human brucellosis in urban and peri-urban areas of Kampala, Uganda. Ann N Y Acad Sci 2008, 1149:309-311.

9. Makita K, Fèvre EM, Waiswa C, Eisler MC, Welburn SC: How human brucellosis incidence in urban Kampala can be reduced most efficiently? A stochastic risk assessment of informally-marketed milk. PLOS ONE 2010, 5(12):e14188.

10. Newton FJ, Jones E, Connor RJ, Davidson BJ, McGovern PT: A survey of bovine brucellosis in four districts of Uganda. Br Vet J 1974, 130:249-254

11. Ndyabahinduka DGK: Brucellosis: an increasing public health hazard in Uganda. Ann Ist Super Sanità 1978, 14:229-234.

12. Oloffs A, Bauman MPO, Afema J, Nakavuma J: Experiences with a strategy to investigate bovine brucellosis in a rural area in Southwest Uganda. Rev Elev Med Vet Pays Trop 1988, 51:101-105.

13. Faye B, Castel V, Lesnoff M, Rutabinda D, Dhalwa J: Tuberculosis and brucellosis prevalence survey on dairy cattle in Mbarara milk basin (Uganda). Prev Vet Med 2005, 67:267-281.

14. Mwiine FN: Benefits and health risks associated with milk and cattle raised in urban and peri-urban areas of Kampala City. Master Thesis Makerere University, Faculty of Veterinary Medicine; 2004.

15. Nakavuma J: Serological survey of Brucella abortus in cattle and goats in the central and southern regions of Uganda. Masters Thesis Makerere University, Kampala; 1994

16. Ndyabahinduka DGK, Chu IH: Brucellosis in Uganda. Int J Zoonoses 1984, 11:59-64.

17. Kalema-Zikusoka G, Bengis RG, Michel AL, Woodford MH: A preliminary investigation of tuberculosis and other diseases in African buffalo (Syncerus caffer) in Queen Elizabeth National Park, Uganda. Onderstepoort J Vet Res 2005, 72:145-151.

18. Kabagambe EK, Elzer PH, Geaghan JP, Opuda-Asibo J, Scholl DT, Miller JE: Risk factors for Brucella seropositivity in goat herds in eastern and western Uganda. Prev Vet Med 2001, 52:91-108.

19. Magona J, Wlubengo J, Galiwango T, Etoori A: Seroprevalence and potential risk of bovine brucellosis in zerograzing and pastoral dairy systems in Uganda. Trop Anim Health Prod 2009, 41:1765-1771.

20. Crawley MJ: Statistical computing: An introduction to data analysis using S-Plus. John-Wiley \& Sons Ltd: Chichester; 2002.

21. Kleinbaum D, Kupper LL, Morgenstein H: Epidemiological Research. Principles and Quantitative measures Lifetime Learning Publications, Belmont; 1982

22. Thrusfield MV: Veterinary epidemiology third edition Blackwell Publishing; 2005.

23. Dohoo I, Martin W, Stryhn H: Veterinary Epidemiologic Research Second Edition The University of Prince Edward Island; 2010.

24. Hosmer DW, Lemeshow S: Applied logistic regression. 2 edition. Toronto, John Wiley \& Sons; 2000.

25. OIE: Manual of Diagnostic Tests and Vaccines for Terrestrial Animals 2008.[http://www.oie.int/eng/normes/mmanual/A_summry.htm].

26. Bundle DR, Gidney MAJ, Duncan JR, Cherwonogrodzky JW: Serological confirmation of Brucella abortus and Yersinia enterocolitica O: 9-antigens by monoclonal antibodies. Infect Immun 1984, 46:389-393.

27. Nielsen K, Duncan JR: Animal Brucellosis Boca Raton: CRC Press; 1990

28. Diaz-Aparicio E, Aragon V, Martin C, Alonso B, Font M, Moreno E, PerezOrtiz S, Blasco JM, Diaz R, Moriyon I: Comparative analysis of Brucella serotype $A$ and $M$ and Yersinia enterocolitica 0:9 polysaccharides for serological diagnosis of brucellosis in cattle, sheep, and goats. J Clin Microbiol 1993, 31:3136-3141.

29. Gall D, Colling A, Marino O, Moreno E, Nielsen K, Perez B, Samartino L: Enzyme immunoassays for serological diagnosis of bovine brucellosis: A trial in Latin America. Clin Diagn Lab Immunol 1998, 5:654-661.

30. Kittelberger R, Bundesen PG, Cloeckaert A, Greiser-Wilke I, Letesson Jj: Serological cross-reactivity between Brucella abortus and Yersinia enterocolitica 0:9: IV. Evaluation of the M- and C-epitope antibody response for the specific detection of B. abortus infections. Vet Microbiol 1998, 60:45-57.

31. Samartino L, Gall D, Gregoret R, Nielsen K: Validation of enzyme-linked immunosorbent assays for the diagnosis of bovine brucellosis. Vet Microbiol 1999, 70:193-200

32. Erdenebaatar J, Bayarsaikhan B, Watarai M, Makino S, Shirahata T: EnzymeLinked Immunosorbent Assay To Differentiate the Antibody Responses of Animals Infected with Brucella Species from Those of Animals Infected with Yersinia enterocolitica 09. 10. Clin Diagn Lab Immunol 2003, 10:710-714.

33. Portanti O, Tittarelli M, Di Febo T, Luciani M, Mercante MT, Conte A, Lelli R: Development and Validation of a Competitive ELISA Kit for the Serological Diagnosis of Ovine, Caprine and Bovine Brucellosis. J Vet Med B Infect Dis Vet Public Health 2006, 53:494-498.

34. Gall D, Nielsen K, Forbes L, Cook W, Leclair D, Balsevicius S, Kelly L, Smith P, Mallory M: Evaluation of the fluorescence polarization assay and comparison to other serological assays for detection of brucellosis in cervids. J Wildl Dis 2001, 37:110-118

35. Nielsen K, Smith P, Yu WL, Halbert G: Salmonella enterica Serotype Urbana Interference with Brucellosis Serology. J Immunoassay Immunochem 2007, 28:289-296.

36. Nielsen K, Smith P, Yu WL, Elmgren C, Nicoletti P, Perez B, Bermudez R, Renteria $\mathrm{T}$ : Second generation competitive enzyme immunoassay for detection of bovine antibody to Brucella abortus. Vet Microbiol 2007. 124:173-177.

37. Kadohira M, McDermott JJ, Shoukri MM, Kyule MN: Variations in the prevalence of antibody to Brucella infection in cattle by farm, area and district in Kenya. Epidemiol Infect 1997, 118:35-41.

38. Abernethy DA, Pfeiffer DU, Watt R, Denny GO, McCullough S: Epidemiology of bovine brucellosis in Northern Ireland between 1990 and 2000. Vet Rec 2006, 158:717-721.

39. Uganda Bureau of Statistics: Statistical Abstract 20052005

40. Nations United: Republic of Uganda, Public Administration Country Profile Division for Public Administration and Development Management (DPADM): Department of Economic and Social Affaires (DESA), United Nations; 2004

41. Scheaffer RL, Mendenhall W III, Ott RL: Elementary Survey Sampling. 5 edition. An Alexander Kugushev Book: Belmont, Duxbury Press; 1996.

42. Makita K, Fèvre EM, Waiswa C, Bronsvoort BMDC, Eisler MC, Welburn SC: Population-dynamics focussed rapid rural mapping and characterisation of the peri-urban interface of Kampala, Uganda. Land Use Policy 2010, 27:888-897.

43. Thrusfield MV, Ortega C, de Blas I, Noordhuizen JP, Frankena K: WINEPISCOPE2.0 Improved epidemiological software for veterinary medicine. Vet Rec 2001, 148:567-572.

44. Gall D, Nielsen K: Serological diagnosis of bovine brucellosis: a review of test performance and cost comparison. Rev Sci Tech 2004, 23:989-1002.

45. van Niekerek A: The effect of body condition as influenced by winter nutrition, on the reproductive performance of the beef cow. S Afr J Anim Sci 1982, 12:383-387.

46. Halekoh U, Højsgaard S, Yan J: The R package geepack for Generalized Estimating Equations. J Stat Softw 2006, 15:1-11.

47. Aragon T: Epidemiology Tools: EpiTools version 0.5-6 R Package for Epidemiologic Data and Graphics; 2010

48. Box GEP, Cox DR: An analysis of transformations. J R Stat Soc B 1964, B 26:211-246.

49. Yongmei N: Link function and distribution diagnostic test for social science researchers: LDdiag 2010.

doi:10.1186/1746-6148-7-60

Cite this article as: Makita et al:: Herd prevalence of bovine brucellosis and analysis of risk factors in cattle in urban and peri-urban areas of the Kampala economic zone, Uganda. BMC Veterinary Research 2011 7:60. 